\title{
A REVIEW OF THE RISK OF DEEP VEIN THROMBOSIS IN SURGICAL AND MINIMALLY INVASIVE TREATMENT OF VARICOSE VEINS
}

\author{
Krzysztof Wołkowski ${ }^{1}$, Tomasz Urbanek ${ }^{2,3}$ \\ 'Department of Surgery, Saint Ann's Hospital, Miechów, Poland \\ 'European Centre of Phlebology, Katowice, Poland \\ ${ }^{3}$ Department of General Surgery, Vascular Surgery, Angiology, and Phlebology, \\ Medical University of Silesia, Katowice, Poland
}

\begin{abstract}
Deep vein thrombosis (DVT) remains one of the most important clinical complications of varicose vein surgery. Despite the fact that procedures employed in the treatment of varicose veins are commonly classified as carrying a low risk of thromboembolism, this risk may vary significantly across different patients due to the presence of individual risk factors. This is a review of the risk of DVT in surgical and minimally invasive treatment of varicose veins. In retrospective studies addressing open varicose vein surgery, the rate of symptomatic DVT has been estimated at approximately $1 \%$, but in prospective imaging-based studies, the risk of DVT in clinical settings seems to be much higher.

The role of risk factors for DVT as well as their proper evaluation and individual risk stratification are significant. Because of population aging, a higher incidence of thromboembolic complications can be expected, suggesting the need for an individualised approach to risk stratification and appropriate risk-adjusted prophylaxis in patients who are candidates for varicose vein treatment. The implementation of new treatment modalities, in particular thermal and non-thermal ablation, does not eliminate the risk of DVT completely despite their relatively minimally invasive nature. Moreover, these methods can be related to the occurrence of new types of thrombotic complications, such as endovenous heat-induced thrombosis. The limited number of prospective studies regarding thromboembolism prophylaxis in modern phlebological treatment warrants further research on the importance of patient assessment and evaluation of procedure-related risk factors for venous thromboembolism.
\end{abstract}

Key words: varicose veins, surgery, endovenous ablation, deep vein thrombosis, prophylaxis.

\author{
REVIEW PAPER \\ Phlebological Review 2018; 26, 2: 44-53 \\ DOl: https://doi.org/10.5114/pr.2018.81012
}

Submitted: 4.08 .2018

Accepted: 10.10 .2018

\author{
ADDRESS FOR CORRESPONDENCE \\ Krzysztof Wołkowski \\ Department of Surgery \\ Saint Ann's Hospital \\ 140 Żeromskiego St. \\ 32-200 Miechów, Poland \\ e-mail: k.wolkowski@poczta.onet.pl
}

\section{INTRODUCTION}

The large number of patients suffering from chronic venous disease (CVD) and technological progress in the treatment of venous incompetence have resulted in a continuously increasing number of patients diagnosed with, and treated for, CVD in western countries [1]. This is a review of the risk of deep vein thrombosis (DVT) after surgical and minimally invasive treatment of varicose veins. The factors influencing this increase include the implementation of new treatment methods, a growing number of dedicated vein treatment centres, and growing awareness regarding CVD diagnosis and treatment among patients and physicians [2,3]. Additionally, the presence of lower leg varicose veins is not only a health problem but also a cosmetic concern for many patients [4].
DVT remains one of the most important clinical complications of varicose vein surgery and other kinds of invasive treatment for superficial vein incompetence [5-8]. Despite the fact that procedures employed in the treatment of varicose veins are commonly classified as carrying a low risk of thromboembolism, this risk may vary significantly across different patients due to the presence of individual risk factors [9]. An increasing prevalence of varicose veins associated with more advanced age and advanced age of the population in general have led to an increase in the number of procedures performed in older patients. In this group and in some younger patients, many concomitant risk factors for venous thromboembolism (VTE) can be identified $[9,10]$. 
According to the current knowledge, many risk factors for VTE are not only treatment-related (such as surgery and its duration, immobilisation, and anaesthesia) but also patient-related (such as age, previous history of VTE, cancer, thrombophilia, inflammatory bowel diseases, acute infections, and many other conditions) [9, 10]. Various clinical advancements in chronic venous disease, different treatment models with their varying durations and invasiveness, as well as different interpatient VTE risk factors suggest the need for an individualised approach to VTE prophylaxis in patients undergoing invasive treatment of varicose veins. In the ENDORSE study (published in 2008) on the practical implementation of VTE prophylaxis in hospitalised patients undergoing medical treatment and surgery, appropriate antithrombotic prophylaxis was used in only $66 \%$ of surgical patients at risk for VTE [11]. Unfortunately, data regarding VTE prophylaxis for varicose vein surgery are not available for this population. The lack of homogeneity between varicose vein populations (in terms of treatment modalities, treatment extent and the presence of VTE risk factors) creates a significant clinical challenge in proper risk stratification and implementation of adequate prophylaxis. Despite some provnposed protocols for the standardisation of VTE prophylaxis in this group of patients, individual risk assessment and implementation of risk-adjusted prophylaxis seem more reasonable $[9,10]$.

\section{DEEP VEIN THROMBOSIS AND VENOUS THROMBOEMBOLISM INCIDENCE IN PATIENTS UNDERGOING VARICOSE VEIN SURGERY AND MINIMALLY INVASIVE TREATMENT (THERMAL ABLATION)}

The occurrence of clinically symptomatic VTE after varicose vein surgery and its potential influence on procedural mortality and morbidity are low in retrospective studies [12, 13]. However, the significance of VTE as a major potentially avoidable cause of in-hospital death in both surgical and medical patients seems unquestionable [10].

The rate of VTE associated with invasive treatment of varicose veins differs significantly across studies $[7,8$, 12-16]. The nature of the study (retrospective or prospective) and methods of assessment (ultrasound or clinical evaluation of symptoms only) are reasons for these significant discrepancies. In most of the retrospective analyses, the reported rate of VTE after varicose vein surgery is approximately $1 \%$ or lower and applies mostly to clinically symptomatic cases [12-16]. In a retrospective analysis of the outcomes of 3300 surgical varicose vein operations, Hagmuller found that diagnoses of clinically symptomatic DVT and pulmonary embolism (PE) were established in $0.15 \%$ and $0.06 \%$ of cases, respectively
[14]. Miller identified DVT episodes in $0.7 \%$ of cases (reduced after the use of antiembolism stockings) in a group of 997 patients who underwent varicose vein surgery [15]. Moreover, Critchley analysed the results of varicose vein surgery on 973 limbs and found that DVT was present in $0.5 \%$ of cases (no PE episodes were reported) [16]. In another retrospective analysis of a large cohort of 1063 patients treated surgically for varicose veins, Huber and Bounameux observed clinical manifestation and confirmed pulmonary embolism in $0.56 \%$ of cases [17]. In a prospective evaluation of the results from a group in which only $3.7 \%$ of patients received pharmacological prophylaxis of VTE, Lemasle et al. reported 17 DVT episodes after 4206 varicose vein operations $(0.4 \%)$ [18]. In this study, only symptomatic patients had an ultrasound scan performed to confirm the DVT diagnosis. Also, one case of PE was noted $(0.02 \%)$ in this group. Moreover, the authors found that the risk for VTE was increased after surgery on the small saphenous vein (three times greater than for the GSV) and after re-do surgeries (three times greater than for primary interventions). Most DVT cases involved calf veins $(71 \%)$, and four occurred in patients who received antithrombotic prophylaxis (out of a total of 155 patients who received pharmacological prophylaxis) [18]. Oesch analysed the results of 8639 vein operations on 12,724 limbs and identified clinically symptomatic VTE episodes in $0.38 \%$ of patients and $0.26 \%$ of operated legs (22 DVT and 14 PE episodes). With the exception of low-risk ambulatory phlebectomy cases, antithrombotic prophylaxis was administered to all patients for 1 to 10 days. In an imaging examination performed to confirm DVT, there were no cases of DVT in the proximal thigh, but seven popliteal vein and 15 crural vein events (distal DVT) were identified. As for the type of the surgery, the highest DVT rate $(1 \%)$ was related to surgery of the small saphenous vein, whereas lower rates were reported for great saphenous vein surgery $(0.46 \%)$ and ambulatory phlebectomy (0.07\%) [19]. In a study based on postoperative duplex imaging of 411 patients who underwent varicose vein surgery, Bhogal and Nyamekye reported a 1.2\% incidence of DVT [20].

Some prospective studies, in particular those based on objective imaging evaluations, report higher DVT rates. In Van Rij's prospective assessment based on Doppler evaluation of 377 patients (performed before and 2-4 weeks after varicose vein surgery, and again at 6-12 months), 5.3\% of DVT cases and no PE episodes were identified [7]. In this study, selective VTE prophylaxis involved a single dose of subcutaneous heparin. Of 20 patients with DVT, only eight were symptomatic ( $1.6 \%$ of patients in the entire population), and 18 cases involved calf veins. Despite a higher DVT rate than previously reported, the clinical significance of most of these events was low. In addition, the procedures in this study were performed under general anaesthesia, and both stripping and phlebectomies were carried out during 
a single surgery. Additionally, as has been mentioned above, pharmacological prophylaxis was used in selected cases only [7]. A higher incidence of DVT after varicose vein treatment, as reported by van Rij, was also suggested by Puttaswamy, based on a duplex US-supported study of 100 lower legs operated for varicose veins. In this study, the reported rate of postoperative DVT was 4.8\% [21].

When considering factors affecting the rate of DVT after varicose vein surgery, not only the study methodology but also the type (phlebectomy, truncal ablation + phlebectomy) and nature (unilateral or bilateral operations) of surgical procedures are significant $[5,13,22,23]$. In a study based on Hospital Episode statistics, Sutton retrospectively analysed VTE episodes occurring within 12 months of varicose vein surgery. The total incidence of VTE was $0.51 \%$, including $0.54 \%$ for open surgery, $0.19 \%$ for sclerotherapy, and $0.47 \%$ for endovenous laser surgery. The incidence of VTE was higher for combined procedures (EVLT + phlebectomies) and amounted to $1.26 \%$. An increased DVT rate was also associated with bilateral treatment (DVT rate: $0.62 \%$ vs. $0.3 \%$ ). Furthermore, re-do surgery and surgery on the short saphenous vein were also associated with increased thrombotic complication rates of $1.62 \%$ and $1.16 \%$, respectively [5]. A potentially higher risk in patients undergoing bilateral varicose vein surgery was also suggested in other studies. For instance, Defty et al. reported $0.14 \%$ of clinically symptomatic DVT cases after single leg operations and $0.75 \%$ in cases of bilateral surgery [22]. Chen et al. investigated the DVT risk in patients undergoing saphenous vein surgical stripping with intraoperative tourniquet application and documented a high rate of DVT. Proximal DVT events occurred in $1.3 \%$ of cases and isolated distal DVT events in $6.4 \%$ of cases [24]. The authors also enumerated DVT risk factors, which were older age ( $\geq 65$ years), female sex, and gastrocnemius vein dilation [24].

Despite the minimally invasive nature of endovenous thermal and non-thermal ablation, VTE has also been encountered after this form of CVD invasive treatment. The number/rate of acute thrombotic episodes varies significantly across reports regarding minimally invasive ablation methods. Considering the possibility of local procedure-related complications, such as EHIT (endovenous heat-induced thrombosis), the reported results should be evaluated carefully, and the true rate of DVT events usually remains low. Jacobs et al. prospectively evaluated the results of 277 consecutive office-based radiofrequency ablations (ClosureFast catheters) and performed duplex ultrasound two weeks after the procedures in all patients $(86 \%$ of the procedures were performed on the great saphenous vein). The DVT, defined by thrombus protrusion with complete occlusion of the femoral or popliteal vein, was visible in only two patients $(0.7 \%)$ [25]. Marsh et al. analysed outcomes after saphenous vein thermal ablation in a group of patients after RF and EVLT. The DVT events occurred in $0.7 \%$ of cases after RF ablation (19 limbs out of 2470 procedures, including four $(0.2 \%)$ EHIT cases) and in $1 \%$ of cases after endovenous laser ablation (four limbs out of 350 procedures, including three $(0.9 \%)$ EHIT cases). In both groups, a low number of thrombotic complications was noted. However, the authors recommended routine postoperative screening until the significance of EHIT, as a local thrombotic complication, is better understood [26]. Knipp et al. compared the rates of symptomatic DVT that had developed in patients after either endovenous laser ablation alone (308 limbs) or after endovenous laser ablation with concomitant phlebectomy or incompetent perforating vein ligation (135 limbs). The DVT events were found in $2.2 \%$ of cases in the combined endovenous and surgical treatment group and in $0 \%$ of cases in the endovenous ablation only group. The rates of saphenofemoral thrombus extension (EHIT) were 5.9\% and $7.8 \%$, respectively. In 181 patients undergoing endovenous laser treatment only, perioperative antithrombotic prophylaxis was used according to a risk stratification protocol. The authors emphasised that, in their cohort, the use of heparin prophylaxis in patients deemed eligible for ablation and phlebectomy did not have a significant effect on the thrombotic complication rate or on thrombus protrusion into deep veins [27]. Dermody et al. analysed the frequency of thromboembolic complications in endovenous thermal ablation and foam sclerotherapy in the treatment of great saphenous vein insufficiency [28]. In the analysis of 12 randomised controlled trials and a 19-case series of endovenous thermal ablation as well as 12 RCTs and a six-case series of foam sclerotherapy, the estimated incidence of VTE was low, $<1 \%$, and was similar across treatment modalities and study types [28].

Benarroch-Gampel et al. searched the database of the national surgical quality improvement program (NSQIP)/2005-2009/ and identified 3874 patients treated by means of saphenous vein radiofrequency ablation $(74.8 \%)$ or laser ablation $(25.2 \%)$ with or without concomitant stab phlebectomy [29]. The incidence of clinically symptomatic DVT was similar in both groups (1.74\% for laser ablation and $1.52 \%$ for radiofrequency ablation). No difference was observed in the occurrence of clinically overt PE: $0.07 \%$ vs. $0 \%$, respectively. The presence of a venous ulcer was identified as a factor that generates a higher DVT rate (2.4-fold higher DVT rate). According to the authors, ensuring proper positioning of the fibre in the perijunction segment and undertaking preventive measures, including VTE anticoagulant prophylaxis, mobilisation, rapid ambulation, and lower extremity compression, are significant, especially in this group (C6 patients) [29]. Based on systematic reviews, cohort studies, and RCTs addressing endovenous laser treatment in more than 10,000 patients, the Ontario Health Technology Advisory Committee estimated the clinically-registered overall pooled major adverse event rate (including DVT, skin burns, or nerve damage) at $0.63 \%$ [30]. How- 
ever, a contrasting and interesting observation comes from a report by O'Donnell et al. [31]. This retrospective analysis involved 131,887 patients diagnosed with, and treated for, varicose veins, including $47.8 \%$ of patients after multiple therapies, $17.4 \%$ after laser ablation, $16.4 \%$ after radiofrequency ablation, 9.6\% after sclerotherapy, and $8.7 \%$ after surgery. The authors followed DVT and PE cases in the medical records of the patients' insurance systems within 30 days of a claim concerning invasive varicose vein treatment. A DVT (expressed as a percentage of events within each treatment cohort) was identified in $4.4 \%$ of the patients treated with radiofrequency thermal ablation, $3.4 \%$ of the patients who underwent multiple therapies on the same day, $3.1 \%$ of the patients who underwent laser ablation, $2.6 \%$ of the patients treated with multiple therapies on different days, $2.4 \%$ of the patients treated with varicose vein surgery, and $0.8 \%$ of sclerotherapy patients. The authors concluded that the rate of thrombotic complications after invasive treatment of varicose veins in the "real world" is probably higher than expected on the basis of small clinical analyses [31].

In light of the anatomical differences between the great and small saphenous veins, some researchers suggest that a DVT rate is higher in patients treated invasively for SSV incompetence. Paravastu et al. analysed the results of endovenous ablation of the small saphenous vein in a Cochrane analysis from 2016 [32]. According to the analysis of three RCTs comparing laser ablation with surgery and one comparing ultrasound-guided foam sclerotherapy (UGFS) with surgery, the rate of DVT was $0.6 \%$ after endovenous laser treatment and $1 \%$ in the group treated with surgery [32].

The minimally invasive nature of thermal and non-thermal ablation is associated with a relatively low rate of DVT, not exceeding $1 \%$ in most reports $[6,23$, 25, 27-29, 31, 33-36]. However, several VTE risk factors identified in patients undergoing this type of procedure can significantly influence the thrombotic event rate. Several other coexisting factors related to the patient's clinical condition and history (including age, a previous DVT episode, or other clinically important factors) can result in an increased procedure risk [21, 23, 25].

Rapid mobilisation and compression (used in most centres early after procedures) can at least theoretically decrease the VTE risk. On the other hand, despite the lack of evidence-based guidelines dedicated to thermal ablation applied for the treatment of varicose veins, individualised risk stratification followed by proper prophylaxis implementation (if required) seem reasonable and clinically justified in this context.

\section{SPECIFIC THROMBOTIC COMPLICATIONS AFTER MINIMALLY INVASIVE TREATMENT}

The introduction of minimally invasive thermal or chemical ablation has elicited the emergence of new thrombotic complications related to the mechanism of ablation (thermal or chemical injury of the venous wall), with possible proximal thrombus propagation (endovenous heat-induced thrombosis - EHIT, endovenous foam-induced thrombosis - EFIT, endovenous glueinduced thrombosis - EGIT) [37-39]. Because the rates of these thrombotic events are significant, their nature, natural history, and potential complications should be investigated and clarified. The incidence of EHIT related to thermal ablation was estimated at $0-16 \%$ for radiofrequency ablation and at $0-7.7 \%$ for laser ablation [40]. The highest EHIT rate was reported by Hingorani et al. in a paper describing the results of the old "closure" radiofrequency system [41]. In this study, based on $66 \mathrm{GSV}$ RF ablation procedures, with $75 \%$ of cases also receiving surgical phlebectomy, DVT was found in 12 limbs (16\%), including 11 cases of EHIT and one case of crural DVT (without significant clinical consequences) [38]. Further studies with new probes and generators do not confirm this high rate of EHIT after thermal ablation. Chi et al. studied 360 consecutive patients after endovenous laser ablation in a prospective ultrasound-supported study and found 19 DVT cases (5.2\%) in the follow-up period, 18 of which involved either the saphenofemoral or saphenopopliteal junction and should be classified as EHIT (DVT independent of the junction area was found in only one case) [36]. The risk factors affecting the thrombotic event rate in this study included age $>66$ years, female gender, and a prior history of superficial vein thrombosis. Sufian et al. investigated risk factors influencing the occurrence of EHIT in patients undergoing radiofrequency ablation [37]. In 6707 treated limbs the incidence of EHIT was 3\%. The identified risk factors for the higher EHIT rate were: large vein diameter, male sex and concomitant multiple phlebectomies [37]. Kane et al. retrospectively analysed data regarding endovenous laser procedures performed on 528 veins (GSV 486; SSV 22) and found EHIT in 5.1\% of cases, including two patients with EHIT in the popliteal vein after small saphenous vein ablation. All cases resolved completely, and no PE was reported after observation or anticoagulation (depending on EHIT advancement) [42]. According to another research study based on 277 consecutive office-based radiofrequency procedures ( $86 \%$ involving the GSV) followed by Doppler US performed within two weeks of the procedures, EHIT was recognised in $4 \%$ of cases and DVT (as ostial thrombus protrusion) in $0.7 \%$ of cases. Previous DVT was the only factor associated with post-procedural DVT. The EHIT occurred more frequently in male patients with factor $\mathrm{V}$ Leiden deficiency, who underwent small saphenous vein ablation [42].

Currently, two classifications of EHIT are available $[38,43]$. According to the one proposed by Kabnick, class I involves saphenous vein thrombosis that develops towards the junction between the superficial and deep 
venous systems (saphenofemoral, saphenopopliteal) but does not extend into the deep venous system; class II is characterised by non-occlusive venous thrombosis, with less than $50 \%$ extension (in a cross-sectional area) into the deep system; class III is characterised by non-occlusive venous thrombosis, with more than $50 \%$ extension into the deep system; class IV includes cases of occlusive DVT of the femoral/popliteal veins [43]. Lawrence et al. proposed a slightly modified and more detailed EHIT classification system based on six stages: class $1-$ a thrombus below the superficial epigastric vein, class 2 - a thrombus at the level of the superficial epigastric vein, class 3 - a thrombus at the level of the femoral vein, class $4-$ a thrombus bulging into the femoral vein, class 5 - a thrombus beyond the saphenopopliteal junction adjacent to the wall of the common femoral vein, and class 6 - femoral vein thrombosis [38].

Because most cases of EHIT remain asymptomatic with a very low rate of progression, heat-induced thrombosis seems to be different from typical superficial thromboses unrelated to invasive treatment. The natural history of endovenous heat-induced thrombosis and the possibility of local thrombus retraction, which is supported by clinical observations of EHIT patients, are the basis for the suggestion that thrombotic sequelae of thermal ablation should be subject to treatment. By contrast with ostial thrombus unrelated to endovenous procedures, thermal injury to the venous wall and heat-induced thrombosis make the thrombus more stable. According to Kabnick, clinical and ultrasound-based follow-up without pharmacological treatment should be proposed for class I EHIT. In class II EHIT, the administration of LMWH with ultrasound control until the thrombus resolves to class I EHIT (usually 10-14 days) is suggested. When class 3 or 4 EHIT is identified, therapeutic anticoagulation is recommended [38, 42-44]. Short-term LMWH anticoagulation with repeated ultrasound scans and follow-up until thrombus regression to class I are proposed in certain centres and in class III EHIT (for class IV EHIT, a therapeutic DVT regimen is suggested) due to the possibility of thrombus retraction and regression. The above-mentioned management guidelines adapted for the Lawrence classification are as follows: for classes 1 and 2 only observation and US follow-up are suggested; for class 3 (thrombus up to the level of the femoral venous wall) a decision should be made according to the surgeon's judgement, including the selection of anticoagulant (LMWH) treatment; for classes 4 and 5 anticoagulation with LMWH is proposed; and for class 6 a full therapeutic DVT treatment regimen is recommended [38]. According to the published outcomes of the clinical implementation of the above-mentioned EHIT management protocol, the stable nature of a thrombus, and low progression and embolisation rates allow safe treatment of most patients [38, 42-45]. Korepta et al. identified 70 patients with EHIT in a study of 4799 ablation procedures. The EHIT patients were treat- ed with aspirin (Kabnick classes I and II) and anticoagulation (Kabnick classes III and IV). In the assessed cohort, no thrombus progression in classes III or IV was found upon a repeat US examination. Simultaneously, thrombus (EHIT) progression was evident in two patients with classes I and II (3\%) [44]. The low rate of EHIT progression is in line with findings reported by other authors. Sufian et al. noted EHIT progression in $4.5 \%$ of cases during follow-up of 201 patients, all of which resolved within four weeks (the authors used anticoagulation in classes III and IV; cases of class I and II EHIT underwent observation). The factors affecting EHIT occurrence in this study were a large vein, male sex, and simultaneous multiple phlebectomies [37].

Other potential EHIT risk factors have been identified in the literature as well. Some of the most commonly suggested are the size (diameter) of the treated vein, proper procedure execution with an appropriate distance between the device tip and the SFJ, and preservation of the vein washing mechanism (related to the preservation of the patency in the superficial epigastric vein and the restoration of blood inflow into the saphenous vein) [23, 29, 37, 46, 47].

Sermsathanasawadi et al. have suggested that a vein diameter $>10 \mathrm{~mm}$, operative time $>40 \mathrm{~min}$, and a Caprini score $>6$ may contribute to a higher EHIT rate in patients treated with radiofrequency ablation [46]. In the study of Rhee et al. EHIT was identified in $4 \%$ of cases, including $6.4 \%$ after endovenous laser ablation and $2.1 \%$ after radiofrequency ablation [48]. Male gender, CEAP classes 3-6, a history of prior thrombosis, and Caprini DVT risk assessment score were also identified as risk factors for EHIT (in the multivariate analysis, Caprini score and male gender remained significant). Jacobs et al. reported EHIT events with thrombus protrusion into the deep system without deep vein occlusion in $4 \%$ of cases treated with endovenous ablation [25]. The only identified factor associated with postprocedural DVT was a previous DVT episode. The factors increasing the risk of EHIT were male sex, SSV treatment, aspirin use, and factor V Leiden deficiency [25].

Hicks et al. analysed the role of concomitant phlebectomy and other potential risk factors for EHIT [23]. In a group of 299 patients who underwent radiofrequency ablation ( $71 \%$ of patients also underwent concomitant mini-phlebectomies), an EHIT was identified in $12 \%$ of cases (including class 1 EHIT in 5\%, class 2 in $4 \%$, class 3 in $1 \%$, and class 4 in $2 \%$ of cases). The incidence of EHIT was much higher after concomitant mini-phlebectomy $(14 \%$ vs. $6 \%)$. In the multivariate analysis, concomitant stab phlebectomy and a history of VTE were independently associated with EHIT [23]. Another study suggested pre-treatment coagulation, assessed by D-dimer measurement in patients undergoing radiofrequency ablation of the great saphenous vein [49]. The authors 
confirmed a higher rate of thrombotic complications in patients with preoperative elevated D-Dimer levels.

Considering a significant number of patients treated with endovenous thermal ablation of the small saphenous vein, EHIT events may occur in this anatomical location. Taking into account the results of research studies, Harlander-Locke et al. proposed an EHIT classification system dedicated to the small saphenous and popliteal vein anatomy [50]. According to this classification, class A describes cases of thrombus extension at least $1 \mathrm{~cm}$ distally from the popliteal vein, class $\mathrm{B}$ defines thrombi at the level of the popliteal vein, class $C$ reflects thrombus extension beyond the popliteal vein, and class D represents totally occlusive DVT of the popliteal vein. When applying this classification to a cohort of RF-treated patients with small saphenous vein incompetence, Harlander-Locke et al. found a thrombus extending into the popliteal vein (class C) in 3\% of the patients. In all cases, thrombus retracted to the level of the saphenopopliteal junction after a short course of anticoagulation (none of the patients developed DVT - class D). The authors of the classification suggest observation as well as clinical and ultrasound-supported follow-up in cases of small saphenous vein EHIT of classes A and B. In patients with class C EHIT, the surgeon's judgement should be followed (anticoagulation or observation). In patients with class D EHIT, therapeutic anticoagulation is recommended $[50,51]$.

Considering the number of performed procedures, the natural history of EHIT, especially in class I and class II, and low progression and embolisation rates reported in the literature, an interesting discussion can arise regarding cost effectiveness of routine post-procedural ultrasound examinations of the lower leg venous system. Since the initial introduction of thermal ablation, mandatory post-procedural ultrasound examinations have been performed in most centres (usually within the first weeks after the procedure).

The main goals of ultrasound are to confirm venous occlusion and identify EHIT. Some researchers question this approach because of the generally unknown time of EHIT occurrence and the unclear clinical significance of the findings relative to the significant health care costs. Ryer et al. investigated 842 patients who underwent great saphenous vein endovenous thermal ablation. All patients were examined by US one day after the procedure, and $79 \%$ of patients had the second examination performed one week after the procedure [52]. The EHIT events were more likely in patients with prior DVT and large great saphenous veins, with $5.1 \%$ of cases requiring anticoagulant treatment. Interestingly, only $47 \%$ of EHIT cases were visible in the initial US examination performed 24 hours after the procedure, and $44 \%$ of EHIT cases were not identified in the first examination but progressed until EHIT was visible on the second US scan. Additionally, late DVT was identified in four patients
(9\%). All of them were diagnosed in another hospital and were not identified during the routine examination protocol. Based on a United States health care pricelist from 2014, the two-ultrasound surveillance algorithm was associated with significant health care charges of 3109 US dollars per delayed VTE event identified. In the conclusion of this study, the authors emphasised the high cost of the EHIT surveillance program and suggested the need for further research to establish cost-effective care for patients after thermal ablation treatment [52].

Because the natural history of EHIT is poorly defined and does not seem to be comparable to unprovoked large superficial vein thrombosis, other authors also question the necessity of routine US examinations after endovenous thermal ablation. In an analysis of endovenous ablation of 13,845 treated limbs, Suarez et al. calculated the number of tests (ultrasound scans) required to detect one case of EHIT/DVT and the total cost of VTE detection according to Medicare fees in the United States of America. The estimated cost related to one case of ultrasound-based VTE detection was 14,667 US dollars. The authors concluded that the low incidence of EHIT and DVT, the unclear clinical significance of ultrasound findings, the mostly benign course of endovenous heat-induced thrombosis, and the high cost of detection of each acute case of thrombosis in routine screening should incite revision of the universal post-EVA screening policy [53].

There is no standardised follow-up protocol dedicated to patients with EHIT or DVT after phlebectomies and minimally invasive treatment. In most reported cases, the diagnosis of DVT has been based on clinical assessment. In patients undergoing open varicose vein procedures, a DVT rate of $0.15 \%$ to $5.3 \%$ can be expected, which is much higher than that reported in prospective ultrasound-based follow-up after varicose vein surgery [7]. The reported rate of DVT in patients undergoing thermal ablation does not appear to exceed $1 \%$, but the above-mentioned thrombotic complications (EHIT) should be taken into account. Considering the low rate of clinically significant DVT, a routine US screening protocol is probably not justifiable from clinical and economical points of view in varicose vein patients undergoing open surgical treatment. The general risk of DVT in patients after thermal ablation seems to be even lower, but some thrombotic cases can be related to procedure-specific complications, such as EHIT, thus justifying the postoperative US-based approach, at least theoretically. The policy of routine postoperative US screening in patients undergoing thermal ablation is now questioned by many researchers due to the mostly benign nature of EHIT, its low clinical significance, low rate of progression, and significant costs of such a protocol. In most patients, EHIT progression to clinically significant VTE is not expected. Further research focussing on EHIT and preoperative DVT risk factors is needed to proper- 
ly identify patients who will require the postprocedural US screening. Some of these factors have already been identified in studies, but the limited knowledge regarding EHIT and its potential sequelae warrants further research in this field, to develop an effective screening protocol.

\section{VENOUS THROMBOEMBOLISM PROPHYLAXIS AFTER VARICOSE VEIN SURGERY AND THERMAL ABLATION}

Despite the reports about thrombotic complications after varicose vein surgery and minimally invasive procedures, a properly defined protocol for VTE prophylaxis is still needed. The variety of procedures, variable disease progression, differences in patient-related VTE risk factors, the type of anaesthesia, and ambulation time render this patient population very non-homogeneous. Other significant problems related to the development of a VTE prophylaxis protocol involve the lack of an appropriate VTE risk evaluation model dedicated to varicose vein patients treated invasively and the limited number of clinical studies on VTE prophylaxis in this population. According to the ACCP guidelines, risk evaluation should be performed in all patients undergoing invasive and non-invasive procedures, and this risk evaluation should be followed by appropriate antithrombotic prophylaxis (adjusted to the estimated VTE risk) [54]. As mentioned before, risk evaluation should concern not only the treatment modality but also patient-dependent risk factors. According to the ACCP document, there is no need for routine thromboprophylaxis in vascular surgery patients, but this does not exclude the need for antithrombotic prophylaxis in patients with clinically significant VTE risk factors $[10,54]$. In the ACCP document and in most other guidelines, no special protocols or advice concerning varicose vein patients can be found [54]. According to the ACCP guidelines, low-risk, moderate-risk, and even high-risk and very high-risk general surgery patients are candidates for prophylaxis. Most varicose vein procedures are considered low- or moderate-risk surgeries, but in some cases, VTE risk factors can significantly elevate this risk, categorising patients into high-risk and even very high-risk groups. Properly defined risk stratification is therefore crucial for sufficient risk evaluation. Unfortunately, no clinically validated protocol dedicated to varicose vein patients undergoing chronic venous disease treatment has been established. The Caprini scale, which is commonly used for surgical patients, has only been validated in some populations, such as intensive care patients, surgical patients and those undergoing plastic surgery [54-57], but not for patients undergoing varicose vein surgery or receiving minimally invasive treatment for chronic venous disease. As mentioned before, according to the study of Rhee et al., the Caprini risk score should be considered in the EHIT risk assessment [48]. In this study on thermal ablation, an ele- vated Caprini score reflected higher likelihood of EHIT occurrence. Considering this result, further research on Caprini score validation and the implementation of other population-specific scales seems clinically justifiable and urgently needed. For an optimal prophylaxis algorithm in patients undergoing varicose vein surgery and minimally invasive treatment, individualised VTE risk stratification should be proposed. Despite the above-mentioned difficulties of objective risk evaluation, both the procedureand patient-related factors should be considered. The short duration and often minimally invasive character of procedures, rapid mobilisation and ambulation, and the use of compression represent significant factors that may contribute to a low VTE risk in the absence of other significant VTE risk factors. In other patients, including those requiring extended surgical intervention, general or spinal anaesthesia, or in those with thrombosis risk factors, immobilised and with comorbidities, a significantly higher risk of VTE can be expected, and the assessment of these cases should be individualised.

The number and quality of studies addressing invasive treatment of varicose veins and focusing on pharmacological thromboprophylaxis are very limited, and their results are inconclusive. In a randomised controlled trial, San Norberto García et al. prospectively randomised 262 moderate-risk patients undergoing varicose vein surgery to a group receiving LMWH (bemiparin) at a prophylactic dose for 10 days postoperatively with other standard methods (compression, early ambulation) or to a group managed in the standard way only [58]. The primary study endpoint was the composite of DVT (symptomatic or asymptomatic) detected by bilateral duplex scanning. In the study, no cases of DVT and no clinical cases of PE, death, or major bleeding were found in any of the groups. The authors suggested that the LMWH routine for perioperative DVT prophylaxis in moderate-risk patients undergoing varicose vein surgery and in patients with postoperative compression and early ambulation is unnecessary [58]. Enoch et al. evaluated a group of 2186 patients with no prior history of VTE episodes, undergoing varicose vein surgery. The authors adjusted pharmacological prophylaxis to the presence of DVT risk factors. A total of four clinically symptomatic VTE episodes occurred in 1283 patients receiving prophylaxis due to pre-existing risk factors [59]. In a group of 2196 patients undergoing high ligation and stripping of the GSV, Wang used four different VTE prophylaxis approaches: A - no pharmacological VTE prophylaxis, B - subcutaneous low-dose unfractionated heparin (LDUH) in three divided doses, C - LMWH 6000 IU once daily, and D - LMWH 4000 IU twice daily. The postoperative DVT and PE incidence was significantly higher in group A (DVT 5.17\%, PE $1.48 \%)$ compared to groups B $(0.56 \%, 0 \%), C(0.35 \%$, $0 \%)$, and $\mathrm{D}(0.36 \%, 0 \%)$. No differences were observed between groups B, C, and D in terms of DVT occurrence. The incidence of haemorrhagic complications was gener- 
ally low in each group but was higher in group B $(0.75 \%)$ compared to the other groups. The authors concluded that postoperative VTE thromboprophylaxis following high ligation and GSV stripping effectively reduces venous thrombosis complications [60]. In another prospective randomised study, 48 women received LMWH prophylaxis two days after surgery and 45 women received LMWH within seven days of surgery [61]. However, no significant difference in DVT rates was observed (five DVT cases documented in US $-4.65 \%$, including two in the short prophylaxis group and three in the prolonged prophylaxis group). Despite prolonged prophylaxis, no improvement in the DVT rate was found [61].

A protocol to decrease the risk and occurrence of DVT after varicose vein surgery was also proposed by Frings et al. [62]. It included a reduction in the duration of the procedure to 45 minutes, limitation in the use of local anaesthesia, rapid mobilisation, and the use of heparin/LMWH [62]. If more time is required to complete a procedure, then a staged approach should be used, with the second procedure performed one day after the initial procedure or within two days of the initial procedure. All 2805 patients were imaged before discharge and 79\% returned for follow-up after three months, but only one patient $(0.03 \%)$ presented with femoropopliteal DVT.

To summarise the data regarding perioperative prophylaxis in patients undergoing varicose vein surgery, no consensus exists in terms of indications or the duration for pharmacological prophylaxis in this clinical setting. As mentioned before, the very non-homogeneous patient group in terms of the type of procedure and individual VTE risk factors, as well as the lack of properly designed trials, do not allow final conclusions to be drawn concerning a proper prophylaxis protocol. Similarly, minimally invasive treatment of varicose veins, including thermal ablation, does not have a dedicated follow-up protocol for the same reasons. Patients at risk for VTE should receive active antithrombotic pharmacological prophylaxis, but further research in this field is urgently needed. In most cases, an individualised approach with an individual risk assessment should be proposed, but there is probably no need for routine and obligatory pharmacological prophylaxis in all patients undergoing varicose vein treatment. However, patients at risk for VTE should be candidates for active antithrombotic prophylaxis based on general rules adjusted to the VTE risk level as proposed in the ACCP document and in other guidelines. Of course, rapid mobilisation, ambulation, and compression remain the most important mechanical antithrombotic prophylaxis measures for varicose vein patients. Due to the lack of prospective studies with new anticoagulant drugs in this setting, LMWH remains the drug of choice for pharmacological prophylaxis in this group. Some confirmation of this proposed approach can be found in the literature. In a results survey performed among members of the vascular surgical society of Great Britain and Ireland,
Campbell and Ridler described the routine use of pharmacological prophylaxis of thromboembolism in $12 \%$ of all cases, but prophylaxis implementation based on risk stratification was reported in $71 \%$ of cases [13]. Shabbir et al. performed a study among consultant general surgeons in Ireland regarding LMWH prophylaxis in outpatient surgery, including patients undergoing varicose vein surgery. Fifty-nine per cent of all respondents reported that they stratified patients according to individual VTE risk [63].

\section{CONCLUSIONS}

Despite the minimally invasive nature of varicose vein treatment for chronic venous disease, patients are still at risk of VTE. Due to the lack of properly designed clinical trials and non-homogeneity of patients undergoing surgical and minimally invasive treatment of varicose veins, individual VTE risk-adjusted thromboprophylaxis regimens, together with early mobilisation and ambulation, should be proposed.

\section{The authors declare no conflict of interest.}

\section{References}

1. Jawien A, Grzela T, Ochwat A. Prevalence of chronic venous insufficiency (CVI) in men and women of Poland. Multicenter cross-sectional study of 40095 patients. Phlebology 2003; 18: 110-122.

2. Kiguchi MM, Dillavou ED. Thermal and Nonthermal Endovenous Ablation Options for Treatment of Superficial Venous Insufficiency. Surg Clin North Am 2018; 98: 385-400.

3. DePopas E, Brown M. Varicose Veins and Lower Extremity Venous Insufficiency. Semin Intervent Radiol 2018; 35: 56-61.

4. Urbanek T, Dorobisz A, Gabriel M, Hendiger W, Jawień A, Kucharzewski M, Madycki G, Rybak Z, Staszkiewicz W, Zubilewicz T, Ziaja K. Assessment of public awareness in the field of epidemiology, prevention and treatment of chronic venous diseases in Poland. Phlebological Review 2015; 23: 45-53.

5. Sutton PA, El-Duhwaib Y, Dyer J, Guy AJ. The incidence of post-operative venous thromboembolism in patients undergoing varicose vein surgery recorded in Hospital Episode Statistics. Ann R Coll Surg Engl 2012; 94: 481-483.

6. Carruthers TN, Farber A, Rybin D, Doros G, Eslami MH. Interventions on the superficial venous system for chronic venous insufficiency by surgeons in the modern era: an analysis of ACS-NSQIP. Vasc Endovascular Surg 2014; 48: 482-490.

7. Van Rij AM, Chai J, Hill GB, Christie RA. Incidence of deep vein thrombosis after varicose vein surgery. Br J Surg 2004; 91: 1582-1585.

8. Bounameux H, Hubner O. Postoperative deep vein thrombosis and surgery for varicose veins. BMJ 1996; 312: 1158.

9. Tomkowski W, Kuca P, Urbanek T, Chmielewski D, Krasiński Z, Pruszczyk P, Windyga J, Oszkinis G, Jawień A, Burakowski J, Dybowska M, Kęsik J, Zubilewicz T. Venous thromboembolism - recommendations on the prevention, diagnostic approach and management. The 2017 Polish Consensus Statement. Acta Angiol 2017; 23: 73-113. 
10. Zawilska K, Zawilska K, Bała MM, Błędowski P, Chmielewski DW, Dobrowolski Z, Frączek M, Frołow M, Gajewski P, Guzik T, Jaeschke R, Korman T, Kotarski J, Kozubski W, Krawczyk M, Kruszewski W, Kulikowski J, Kutaj-Wąsikowska H, Mayzner-Zawadzka E, Mrozikiewicz PM, Musiał J, Niżankowski R, Pasierski T, Poręba R, Tomkowski W, Torbicki A, Undas A, Urbanek T, Wojtukiewicz MZ, Woroń J, Wroński J; Working Group fom the Anticoagulation and Thrombolytic ACCP Conference. Polish guidelines for the prevention and treatment of venous thromboembolism. 2012 update. Pol Arch Med Wewn 2012; 122 (Suppl 2): 3-74.

11. Cohen AT, Tapson VF, Bergmann JF, Goldhaber SZ, Kakkar AK, Deslandes B, Huang W, Zayaruzny M, Emery L, Anderson FA Jr; ENDORSE Investigators. ENDORSE Venous thromboembolism risk and prophylaxis in the acute hospital care setting (ENDORSE study): a multinational cross-sectional study. Lancet 2008 ; 371: 387-394.

12. Enoch $\mathrm{S}$. Incidence of deep vein thrombosis after varicose vein surgery. Br J Surg 2004; 91: 1582-1585.

13. Campbell WB, Ridler BMF. Varicose vein surgery and deep vein thrombosis. Br J Surg 1995; 82: 1494-1497.

14. Hagmuller GW. Complications in surgery of varicose veins. Langenbecks Arch Chir 1992: 470-474.

15. Miller GV, Lewis WG, Sainsbury JR, Macdonald RC. Morbidity of varicose vein surgery: auditing the benefit of changing clinical practice. Ann R Coll Surg Engl 1996; 78: 345-349.

16. Critchley G, Handa A, Maw A, Harvey A, Harvey MR, CorbettCR. Complications of varicose vein surgery. Ann R Coll Surg Engl 1997; 79: 105-110.

17. Huber O, Bounameaux H, Borst F, Rohner A. Postoperative pulmonary embolism after hospital discharge. An underestimated risk. Arch Surg 1992; 127: 310-313.

18. Lemasle P, Lefebvre Vilardebo M, Uhl JF. Should we really prescribe anticoagulants after surgery for excision of varicose veins? Phlébologie 2004; 57: 187-194.

19. Oesch A. Thrombosis and pulmonary embolism risk in patients undergoing varicose vein surgery. Phlebolymphology 2013; 20: 96-100.

20. Bhogal RH, Nyamekye IK. Should all patients undergo postoperative duplex imaging to detect a deep vein thrombosis after varicose vein surgery? World J Surg 2008; 32: 237-240.

21. Puttaswamy V, Fisher C, Applebery M. Venous thromboembolism following varicose vein surgery: a prospective analysis. Aust N Z J Surg 2000; 70 (Suppl): A150.

22. Defty C, Eardley N, Taylor M, Jones DR, Mason PF. A comparison of the complication rates following unilateral and bilateral varicose vein surgery. Eur J Vasc Endovasc Surg 2008; 35: 745-749.

23. Hicks CW, DiBrito SR, Magruder JT, Weaver ML, Barenski C, Heller JA. Radiofrequency ablation with concomitant stab phlebectomy increases risk of endovenous heat-induced thrombosis. J Vasc Surg Venous Lymphat Disord 2017; 5: 200-209.

24. Chen K, Yu GF, Huang JY, Huang LD, Su X, Ni HZ, Pan LM, Zheng XT. Incidence and risk factors of early deep venous thrombosis after varicose vein surgery with routine use of a tourniquet. Thromb Res 2015; 135: 1052-1056.

25. Jacobs CE, Pinzon MM, Orozco J, Hunt PJ, Rivera A, McCarthy WJ. Deep venous thrombosis after saphenous endovenous radiofrequency ablation: is it predictable? Ann Vasc Surg 2014; 28: 679-685.
26. Marsh P, Price BA, Holdstock J, Harrison C, Whiteley MS. Deep vein thrombosis (DVT) after venous thermoablation techniques: rates of endovenous heat-induced thrombosis (EHIT) and classical DVT after radiofrequency and endovenous laser ablation in a single centre. Eur J Vasc Endovasc Surg 2010; 40: 521-527.

27. Knipp BS, Blackburn SA, Bloom JR, Fellows E, LaForge W, Pfeifer JR, Williams DM, Wakefield TW; Michigan Venous Study Group. Endovenous laser ablation: venous outcomes and thrombotic complications are independent of the presence of deep venous insufficiency. J Vasc Surg 2008; 48: 1538-1545.

28. Dermody M, Schul MW, O’Donnell TF. Thromboembolic complications of endovenous thermal ablation and foam sclerotherapy in the treatment of great saphenous vein insufficiency. Phlebology 2015; 30: 357-364.

29. Benarroch-Gampel J, Sheffield KM, Boyd CA, Riall TS, Killewich LA. Analysis of venous thromboembolic events after saphenous ablation. J Vasc Surg Venous Lymphat Disord 2013; 1: 26-32.

30. Health Quality Ontario. Endovascular Laser Therapy for Varicose Veins: An Evidence-Based Analysis. Ont Health Technol Assess Ser 2010; 10: 1-92.

31. O’Donnell TF, Eaddy M, Raju A, Boswell K. Wright DAssessment of thrombotic adverse events and treatment patterns associated with varicose vein treatment. J Vasc Surg Venous Lymphat Disord 2015; 3: 27-34.

32. Paravastu SCV, Horne M, Dodd PD. Endovenous ablation therapy (laser or radiofrequency) or foam sclerotherapy versus conventional surgical repair for short saphenous varicose veins. Cochrane Database Syst Rev 2016; 11: CD010878.

33. Puggioni A, Kalra M, Carmo M, Mozes G, Gloviczki P. Endovenous laser therapy and radiofrequency ablation of the great saphenous vein: analysis of early efficacy and complication. J Vasc Surg 2005; 42: 488-493.

34. Malgor RD, Gasparis AP, Labropoulos N. Morbidity and mortality after thermal venous ablations. Int Angiol 2016; 35: 57-61.

35. Boersma D, Kornmann VN, van Eekeren RR, Tromp E, Ünlü Ç, Reijnen MM, de Vries JP. Treatment Modalities for Small Saphenous Vein Insufficiency: Systematic Review and Meta-analysis. J Endovasc Ther 2016; 23: 199-211.

36. Chi YW, Woods TC. Clinical risk factors to predict deep venous thrombosis post-endovenous laser ablation of saphenous veins. Phlebology 2014; 29: 150-153.

37. Sufian S, Arnez A, Labropoulos N, Lakhanpal S. Incidence, progression, and risk factors for endovenous heat-induced thrombosis after radiofrequency ablation. J Vasc Surg Venous Lymphat Disord 2013; 1: 159-164.

38. Lawrence PF, Chandra A, Wu M, Rigberg D, DeRubertis B, Gelabert H, Jimenez JC, Carter V. Classification of proximal endovenous closure levels and treatment algorithm. J Vasc Surg 2010; 52: 388-393.

39. Kulkarni SR, Messenger DE, Slim FJ, Emerson LG, Bulbulia RA, Whyman MR, Poskitt KR. The incidence and characterization of deep vein thrombosis following ultrasound-guided foam sclerotherapy in 1000 legs with superficial venous reflux. J Vasc Surg Venous Lymphat Disord 2013; 1: 231-238.

40. Mozes G, Kalra M, Carmo M, Swenson L, Gloviczki P. Extension of saphenous thrombus into the femoral vein: a potential complication of new endovenous ablation techniques. J Vasc Surg 2005; 41: 130e. 
41. Hingorani AP, Ascher E, Markevich N, Schutzer RW, Kallakuri S, Hou A, Nahata S, Yorkovich W, Jacob T. Deep venous thrombosis after radiofrequency ablation of greater saphenous vein: a word of caution. J Vasc Surg 2004; 40: 500-504.

42. Kane K, Fisher T, Bennett M, Shutze W Jr, Hicks T, Grimsley B, Gable D, Pearl G, Smith B, Shutze W Sr. The incidence and outcome of endothermal heat-induced thrombosis after endovenous laser ablation. Ann Vasc Surg 2014; 28: 1744-1750.

43. Kabnick LS, Ombrellino M, Agis H, Mortiz M, Almeida J, Baccaglini U, et al. Endovenous heat induced thrombus (EHIT) at the superficial deep venous junction: a new post-treatment clinical entity, classification and potential treatment strategies. 18th Annual Meeting of the American venous Forum, Miami, Florida 2006.

44. Korepta LM, Watson JJ, Mansour MA, Chambers CM, Cuff RF, Slaikeu JD, Wong PY. Outcomes of a single-center experience with classification and treatment of endothermal heat-induced thrombosis after endovenous ablation J Vasc Surg Venous Lymphat Disord 2017; 5: 332-338.

45. Santin BJ, Lohr JM, Panke TW, Neville PM, Felinski MM, Kuhn BA, Recht MH, Muck PE. Venous duplex and pathologic differences in thrombus characteristics between de novo deep vein thrombi and endovenous heat-induced thrombi. J Vasc Surg Venous Lymphat Disord 2015; 3: 184-189.

46. Sermsathanasawadi N, Voravitvet TY, Chinsakchai K, Wongwanit C, Ruangsetakit C, Mutirangura P. Risk factors for endovenous heat-induced thrombosis after endovenous radiofrequency ablation performed in Thailand. Phlebology 2016; 31: 582-587.

47. Ahn S, Jung IM, Chung JK, Lee T. Changes in Saphenous Vein Stump and Low Incidence of Endovenous Heat-Induced Thrombosis After Radiofrequency Ablation of Great Saphenous Vein Incompetence. Dermatol Surg 2016; 42: 515-520.

48. Rhee SJ, Cantelmo NL, Conrad MF, Stoughton J. Factors Influencing the Incidence of Endovenous Heat-Induced Thrombosis (EHIT). Vasc Endovascular Surg 2013; 47: 207-212.

49. Lurie F, Kistner RL. Pretreatment elevated D-dimer levels without systemic inflammatory response are associated with thrombotic complications of thermal ablation of the great saphenous vein. J Vasc Surg Venous Lymphat Disord 2013; 1: 154-158.

50. Harlander-Locke M, Jimenez JC, Lawrence PF, Derubertis BG, Rigberg DA, Gelabert HA, Farley SM. Management of endovenous heat-induced thrombus using a classification system and treatment algorithm following segmental thermal ablation of the small saphenous vein. J Vasc Surg 2013; 58: 427-431.

51. Harlander-Locke M, Jimenez JC, Lawrence PF, Derubertis BG, Rigberg DA, Gelabert HA. Endovenous ablation with concomitant phlebectomy is a safe and effective method of treatment for symptomatic patients with axial reflux and large incompetent tributaries. J Vasc Surg 2013; 58: 166-172.

52. Ryer EJ, Elmore JR, Garvin RP, Cindric MC, Dove JT, Kekulawela S, Franklin DP. Value of delayed duplex ultrasound assessment after endothermal ablation of the great saphenous vein. J Vasc Surg 2016; 64: 446-451.

53. Suarez L, Tangney E, O’Donnell TF, Iafrati MD. Cost analysis and implications of routine deep venous thrombosis duplex ultrasound scanning after endovenous ablation. J Vasc Surg Venous Lymphat Disord 2017; 5: 126-133.
54. Gould MK, Garcia DA, Wren SM, Karanicolas PJ, Arcelus JI, Heit JA, Samama CM. Prevention of VTE in nonorthopedic surgical patients: Antithrombotic Therapy and Prevention of Thrombosis, 9th ed: American College of Chest Physicians Evidence-Based Clinical Practice Guidelines. Chest 2012; 141 : 227-277.

55. Caprini JA. Risk assessment as a guide to thrombosis prophylaxis. Curr Opin Pulm Med 2010; 16: 448-452.

56. Shuman AG, Hu HM, Pannucci CJ, Jackson CR, Bradford CR, Bahl V. Stratifying the risk of venous thromboembolism in otolaryngology. Otolaryngol Head Neck Surg 2012; 146: 719-724.

57. Jeong HS, Miller TJ, Davis K, Matthew A, Lysikowski J, Lazcano E, Reed G, Kenkel JM. Application of the Caprini risk assessment model in evaluation of non-venous thromboembolism complications in plastic and reconstructive surgery patients. Aesthet Surg J 2014; 34: 87-95.

58. San Norberto García EM, Merino B, Taylor JH, Vizcaíno I, Vaquero C. Low-molecular-weight heparin for prevention of venous thromboembolism after varicose vein surgery in moderate-risk patients: a randomized, controlled trial. Ann Vasc Surg 2013; 27: 940-946.

59. Enoch S, Woon E, Blair SD. Thromboprophylaxis can be omitted in selected patients undergoing varicose vein surgery and hernia repair. Br J Surg 2003; 90: 818-820.

60. Wang H, Sun Z, Jiang W, Zhang Y, Li X, Wu Y. Postoperative prophylaxis of venous thromboembolism (VTE) in patients undergoing high ligation and stripping of the great saphenous vein (GSV). Vasc Med 2015; 20: 117-121.

61. Warot M, Synowiec T, Wencel-Warot A, Daroszewski P, Bojar I, Micker M, Chęciński P. Can deep vein thrombosis be predicted after varicose vein operation in women in rural areas? Ann Agric Environ Med 2014; 21: 601-605.

62. Frings N, Glowacki P, Tran P, Schäfer L. Varicose vein surgery Avoiding deep venous thrombosis. Phlebologie 2006; 35: 233236.

63. Shabbir J, Ridgway PF, Shields W, Evoy D, O’Mahony JB, Mealy K. Low molecular weight heparin prophylaxis in day case surgery. Ir J Med Sci 2006; 175: 26-29. 